\title{
Erratum to: Magnetic-nanobead-based competitive enzyme-linked aptamer assay for the analysis of oxytetracycline in food
}

\author{
Chunxia Lu ${ }^{1,2} \cdot$ Zonggui Tang $^{1,2} \cdot$ Changbin Liu $^{3} \cdot$ Lichao Kang $^{1,2} \cdot$ Fengxia Sun ${ }^{1,2}$
}

Received: 19 May 2015 / Accepted: 19 May 2015 / Published online: 29 May 2015

(C) Springer-Verlag Berlin Heidelberg 2015

\section{Erratum to: Anal Bioanal Chem}

DOI 10.1007/s00216-015-8632-3

Regrettably, Fengxia Sun was missing as the second corresponding author for this article.

The correct author list should read as printed above.

Chunxia Lu and Fengxia Sun are both corresponding authors.

\section{Chunxia Lu \\ shzlcx2002@163.com \\ $\triangle$ Fengxia Sun \\ 119772198@qq.com}

1 Analysis and Testing Center, Xinjiang Academy of Agriculture and Reclamation Science, Wuyi Road No. 221, Shihezi 832000, China

2 Supervision and Inspection Testing Center of Foods Quality (Shihezi), Ministry of Agriculture, Wuyi Road No. 221, Shihezi 832000, China

3 Institute of Animal Husbandry and Veterinary Science, Xinjiang Academy of Agriculture and Reclamation Science, Wuyi Road No. 221, Shihezi 832000, China 